\title{
Increased Emergency Department Use by Adolescents and Young Adults with Eating Disorders
}

\author{
Suzanne Dooley-Hash, MD ${ }^{1,2 *}$ \\ Sarah K. Lipson, MEd ${ }^{3}$ \\ Maureen A. Walton, MPH, $\mathrm{PhD}^{4}$ \\ Rebecca M. Cunningham, MD ${ }^{1,5}$
}

\begin{abstract}
Objective: This study describes patterns of emergency department (ED) utilization by patients who screen positive for eating disorders.
\end{abstract}

Method: ED patients aged $14-20$ years ( $n=1,920)$ completed a computerized questionnaire. The analyses compared the rates of ED use between patients who screened positive for an eating disorder and those who did not and examined the reasons for ED use amongst patients with eating disorders.

Results: ED patients who screened positive for eating disorders were significantly more likely to have previously visited the ED and, on average, utilized the

\begin{abstract}
ED at a rate 1.6 times higher than patients who screen negative for eating disorders. The most common chief complaints among patients who screen positive for eating disorders were abdominal pain and other gastrointestinal-related problems.
\end{abstract}

Discussion: Patients with eating disorders utilize the ED more frequently than those without and commonly present for complaints seemingly unrelated to their eating disorder. () 2012 by Wiley Periodicals, Inc.

Keywords: eating disorder; emergency department; utilization

(Int J Eat Disord 2013; 46:308-315)

\section{Introduction}

People with eating disorders utilize healthcare resources at higher rates than the general population. This has been demonstrated in patients across multiple healthcare settings both pre-eating and posteating disorder diagnosis. ${ }^{1-5}$ Prior studies conducted in primary care settings have shown increased frequency of both office visits and telephone contact by patients with eating disorders. ${ }^{1,2}$ These studies have found that patients with eating disorders often present to their healthcare providers with general medical complaints such as fa-

\footnotetext{
Accepted 3 September 2012

Conflict of Interest: There are no conflicts of interest to report for any of the study authors.

Supported by AA018122 from the National Institute on Alcohol Abuse and Alcoholism (NIAAA).

*Correspondence to: Suzanne Dooley-Hash, MD, Department of Emergency Medicine, University of Michigan, Ann Arbor, MI. E-mail:sldh@umich.edu

${ }^{1}$ Department of Emergency Medicine, University of Michigan, Ann Arbor, Michigan

${ }^{2}$ The Center for Eating Disorders, Ann Arbor, Michigan

${ }^{3}$ Center for the Study of Higher and Postsecondary Education, School of Education, University of Michigan, Ann Arbor, Michigan

${ }^{4}$ Department of Psychiatry, University of Michigan, Ann Arbor, Michigan

${ }^{5}$ Department of Emergency Medicine, University of Michigan Injury Center, Ann Arbor, Michigan

Published online 9 October 2012 in Wiley Online Library (wileyonlinelibrary.com). DOI: 10.1002/eat.22070

(C) 2012 Wiley Periodicals, Inc.
}

tigue, abdominal pain, constipation, and amenorrhea, which are potentially related to the disorder but are not recognized as such by the patients or providers. ${ }^{1,2,4,6}$ As a result, patients who are eventually diagnosed with an eating disorder receive more referrals to specialists such as gastroenterologists and gynecologists than other patients ${ }^{1}$ and frequently undergo extensive diagnostic evaluations for an organic cause of their symptoms prior to identification of the underlying eating disorder. ${ }^{7}$ Despite this increased use of resources, the majority of eating disorders go undiagnosed for years. ${ }^{8-10}$

Although fewer studies of healthcare utilization in patients with eating disorders have included acute care settings such as urgent care and emergency departments (EDs), increased use of these services in patients with eating disorder when compared with controls has also been noted. ${ }^{5,9,11}$ Given that the likelihood of successful treatment of eating disorders is inversely related to the duration of untreated illness, early detection is vital. ${ }^{12,13}$ Every visit to a healthcare professional represents an opportunity for identification, which may be facilitated by an improved understanding of patterns of ED use by these patients. A previously published article from the current study group showed that the prevalence of eating disorders was $16 \%$ among youth aged 14-20 years who presented to the ED and that correlates of screening positive for an eating disorder among these patients were gender, body mass index (BMI), risky drinking behavior, 
and depression. ${ }^{14}$ The current article builds on our previous descriptive findings using a larger sample size $(\sim 1,000$ more participants) to examine correlates of increased ED use. Specifically, this study focused on the rates of ED use among patients who screened positive for an eating disorder and the reasons for ED use amongst patients with eating disordered. We hypothesized that patients with eating disorders would have higher rates of ED utilization than those without and that most would present with complaints not directly attributed to an eating disorder.

\section{Method}

\section{Study Design and Setting}

This study used the data collected from ED patients as part of a larger study, Project UConnect, which is conducted at the University of Michigan Medical Center in Ann Arbor, Michigan, a large, academic medical center with a Level 1 trauma designation. The university's institutional review board approved all study activities, and the National Institutes of Health issued a Certificate of Confidentiality for human participants.

Individuals meeting inclusion criteria (patients aged 14-20 years presenting to the ED between October 15, 2010 and September 14, 2011) were eligible to participate. To minimize disruptions for patients in acute psychological distress, those seeking care for suicidal ideation, sexual assault, or child abuse were excluded from participation in the study. Those who did not speak English, were visually or hearing impaired, neurologically impaired such that informed consent could not be obtained, physically combative, in police custody, or aged below 18 years with no parent/guardian available for consent were also excluded. Patients who were admitted to the hospital and had been too ill to participate while in the ED were eligible for approach during their inpatient stay.

Research assistants (RAs) identified potential study participants in patient treatment areas using the ED's electronic whiteboard (patient tracking system). After written consent was obtained (from patients or their parent/ guardian if aged below 18 years) and following instructions on the use of a touchscreen computer, study participants completed the electronic screening survey. Most participants completed the survey in less than $20 \mathrm{~min}$. Participants were given a small financial incentive (a gift valued at \$1.00) following completion of the survey.

A retrospective chart review of each participant's electronic medical record was later completed by RAs. Information recorded included number of ED visits in the 12 months prior to the index visit, the patient's self-reported chief complaint at the index visit, the physician-assigned discharge diagnosis at that visit, and whether the visit was due to a medical complaint or an injury. Regular auditing of medical chart reviews conducted on 5\% of charts using procedures similar to those described by Gilbert and Lowenstein ${ }^{15}$ revealed an error rate of $<2 \%$.

\section{Independent Variables}

Demographics. Demographic information including gender, age, race, and receipt of public assistance was collected. A dichotomous measure of public assistance was drawn from a survey item which asked patients whether their parents or "the most important person in raising you" receive some form of public assistance (e.g., welfare, Bridge Card, EBT, and disability benefits).

Body Mass Index. The participants' self-reported height and weight were used to calculate the BMI using the following formula: $\mathrm{BMI}=$ weight in pounds $/\left(\right.$ height in inches) ${ }^{2} \times$ 703. The patients were classified as underweight (BMI < $18.5)$, normal weight $(\mathrm{BMI}=18.5-24.9)$, overweight $(\mathrm{BMI}=$ 25.0-29.9), and obese (BMI $\geq 30.0$ ) based on the BMI categories defined by the World Health Organization. ${ }^{16}$

Eating Disorders. A series of five "yes" or "no" questions were used to screen for eating disorders: (1) Do you ever make yourself throw up (or use laxatives, water pills, or exercise) because you feel uncomfortably full?; (2) Do you worry you have lost control over how much you eat?; (3) Have you recently lost or gained more than 10-15 pounds in a 3-month period?; (4) Do you believe yourself to be too fat when others say you are too thin?; and (5) Do thoughts and fears about food and weight dominate your life? These questions are based on the SCOFF, which is an empirically validated self-report questionnaire used to screen for eating disorders in the primary care setting. ${ }^{17-20}$ Prior studies have established a cutoff score of $\geq 2$ positive answers to the SCOFF questions and have determined that cutoff to be both sensitive (72-100\%) and specific (73-94\%) for the diagnosis of anorexia nervosa (AN) and/or bulimia nervosa (BN). ${ }^{17-20}$ The original SCOFF questionnaire was developed in the United Kingdom for use in adult populations as a screening instrument for AN and BN only. Participants in the current study were given a modified version of the SCOFF. Modifications were intended to increase understanding of the questions in US sample (e.g., "stone" changed to "pounds") and to capture disordered eating behaviors including but not limited to AN or BN (e.g., binge eating disorder and other forms of eating disorders not otherwise specified). For instance, "gained" was added to Question 3 to evaluate for weight gain and/or cycling rather than just weight loss.

Depression. Symptoms of depression over the past 2 weeks were screened by using the 2-question version of the Patient Health Questionnaire (PHQ-2). Depressive 
symptoms are assessed using a frequency scale of 0 (not at all) through 3 (nearly every day). ${ }^{21}$ A cutoff score of $\geq 3$ on the PHQ-2 was chosen for this study based on prior studies that have shown this score to be effective in screening for major depression. ${ }^{22,23}$

Alcohol Use. The first three questions (consumption items) of the 10-item Alcohol Use Disorders Identification Test (AUDIT-C) were used to measure risky drinking behavior over the last 3 months. Prior studies have established cutoff scores of $<3$ for adolescents aged 14-17 years $^{24}$ and $<4$ for those aged 18-20 years. ${ }^{25}$ These scores for the AUDIT-C were used in this study as they have previously been shown to be both sensitive and specific for identifying risky drinking behavior in these age groups and a good predictor of future alcohol-related social and medical problems. ${ }^{26,27}$

Drug Use. Questions used to screen for tobacco and/or drug use over the past 3 months were taken from the Alcohol, Smoking and Substance Involvement Screening Test. ${ }^{28}$ Substances screened for include over-the-counter and prescription medications as well as illicit substances. The substances were categorized as tobacco, cannabis, stimulants (methamphetamine, cocaine, and prescription stimulants), and other drugs (inhalants, hallucinogens, illicit and prescription opioids, dextromethorphan, and sedatives) and were coded as dichotomous variables (no use/any use) for analyses.

\section{Outcome Measures}

The primary outcome measure of interest was the frequency of ED visits by patients during the 12 months prior to their index visit. For each study participant, RAs reviewed the electronic medical records for the number of visits to the $\mathrm{ED}$ in the 12 months prior to the date of index visit and baseline screening. From these data, we created a dichotomous measure $(0=$ no previous visits; 1 $=$ one or more previous visits) and a count variable (0-14 previous visits). We conducted statistical analyses using both a dichotomous measure and a count variable measure of visits to the ED over the past 12 months.

The secondary outcome measure was the patients' primary reason for their ED visit. This information was taken directly from each patient's self-reported chief complaint as found in the electronic medical record for that visit. These complaints were then coded by an ED physician (S.D.-H.) and categorized as gastrointestinal (GI), neurologic, genitourinary (GU), injury, or other. GI complaints included abdominal pain, nausea, vomiting, diarrhea, constipation, hematemesis, or rectal bleeding. Neurologic complaints included syncope, seizure, weakness, dizziness, lightheadedness, headache, numbness, or tingling. Pregnancy-related complaints, vaginal bleeding or discharge, urinary frequency, urgency, and retention as well as flank and pelvic pain were coded as GU complaints. Injury included trauma from assault, motor vehicle collisions, burns, sports injuries, and falls. Complaints not falling into one of these four complaint categories were categorized as "other." We used these data to create a categorical chief complaint variable $(1=\mathrm{GI} ; 2=$ neurology; 3 = GU; 4 = injury; 5 = other).

\section{Statistical Analysis}

We investigated individual characteristics associated with increased ED use using bivariate analyses and $\chi^{2}$ tests. We estimated negative binomial regression to estimate the impact of key variables on the number of times patients visit the ED over a 12-month period. Here, we report incidence rate ratios and $95 \%$ confidence intervals (CIs). A negative binomial regression was the most appropriate statistical model given that our outcome was an overdispersed count variable, that is, the conditional variance of our dependent variable exceeded its conditional mean. Overdispersion of our outcome variable can be attributed to the fact that the majority of ED patients in our sample had no previous visits to the ED. Of 1,920 patients in our sample, 1,443 had no previous visits to the ED during the 12 months prior to the baseline screening, whereas 477 (33\%) had visited the ED once or more during this time. All statistical analyses were conducted using STATA 12.1 (StataCorp, College Station, TX).

\section{Results}

Of 4,153 patients who presented to the ED during the study period, 2,679 (64.5\%) met inclusion criteria and were eligible for screening. RAs approached 2,225 (83.1\%) for screening and missed 454 (16.9\%). Of the patients approached, $1,922(86.4 \%)$ agreed to participate, whereas $303(13.6 \%)$ refused. Of the study participants, 90 participants did not complete all five SCOFF questions and two participants did not report their age. Baseline characteristics for these 92 participants were not significantly different from those with complete screens and they were excluded from analyses (data not shown).

Our final sample size was 1,920 ED patients aged between 14 and 20 years. The average age of respondents in years was $17.5(\mathrm{SD}=2.0)$. The majority of respondents were female $(56.5 \%)$ and White $(72.2 \%)$. Of these respondents, $24.1 \%$ indicated that their parents/guardians receive public assistance. The average BMI of respondents in our sample was 23.8 ( $\mathrm{SD}=7.0$ ). Of these respondents, $15.5 \%$ screened positive for an eating disorder based on the cutoff score of $\geq 2$ positive answers on the SCOFF. Of those who screened positive for an eating disorder, $77.8 \%$ were female, $24.7 \%$ were 
TABLE 1. Bivariate Analyses of Emergency Department Use in the 12 Months Prior to Index Visit

\begin{tabular}{|c|c|c|c|c|c|}
\hline & $\begin{array}{l}\geq 1 \text { Previous ED Visit } \\
(n=477,24.9 \%)\end{array}$ & $\begin{array}{l}\text { Unadjusted } \\
\text { ORs }\end{array}$ & $\begin{array}{l}\text { Standard } \\
\text { Error }\end{array}$ & $p$-value & $95 \% \mathrm{Cl}$ \\
\hline Age $($ mean $=17.5, \mathrm{SD}=2.0)$ & $17.5(1.9)$ & 1.0 & 0.03 & .7 & $1.0-1.1$ \\
\hline White $(n=1,387,72.2 \%)$ & $326(68.3)$ & 0.8 & 0.1 & .03 & $0.6-1.0$ \\
\hline Public assistance (yes) $(n=460,24.1 \%)$ & $157(33.0)$ & 1.8 & 0.2 & $<.001$ & $1.5-2.3$ \\
\hline Depression (PHQ-2 $\geq 3)(n=262,13.9 \%)$ & $97(20.5)$ & 2.0 & 0.3 & $<.001$ & $1.5-2.6$ \\
\hline $\begin{array}{l}\text { Risky drinking behavior (AUDIT-C } \geq 3 \text { if } 14-17 \text { years; } \\
\quad \geq 4 \text { if } 18-20 \text { years) ( } n=713,37.1 \%)\end{array}$ & $170(35.6)$ & 0.9 & 0.1 & .4 & $0.7-1.1$ \\
\hline Drug use $(n=495,25.8 \%)$ & $147(30.8)$ & 1.4 & 0.2 & .004 & $1.1-1.8$ \\
\hline $\begin{array}{l}\text { Screened positive for eating disorder } \\
\quad(\text { SCOFF } \geq 2)(n=284,15.5 \%)\end{array}$ & $104(22.7)$ & 1.9 & 0.3 & $<.001$ & $1.5-2.5$ \\
\hline
\end{tabular}

TABLE 2. Comparison of Number of ED Visits in the Previous 12 Months Between Patients Who Screen Positive Versus Negative for Eating Disorders $\left(\chi^{2}=38.1, p<.001\right)$

\begin{tabular}{|c|c|c|c|c|c|c|c|}
\hline & \multicolumn{6}{|c|}{ Total Number of Previous ED Visits } & \multirow[b]{2}{*}{ Total } \\
\hline & 0 & 1 & 2 & 3 & 4 & $5+$ & \\
\hline $\begin{array}{l}\text { Screened positive for an eating disorder } \\
\quad(\text { SCOFF } \geq 2)\end{array}$ & $180(12.5 \%)$ & $64(21.2 \%)$ & $14(14.6 \%)$ & $7(25.0 \%)$ & $6(28.6 \%)$ & $13(43.3 \%)$ & 284 \\
\hline No eating disorder $(\mathrm{SCOFF}<2)$ & $1,190(82.5 \%)$ & $226(74.8 \%)$ & $77(80.2 \%)$ & $20(71.4 \%)$ & $15(71.4 \%)$ & $17(56.7 \%)$ & 1,545 \\
\hline Total & 1370 & 290 & 91 & 27 & 21 & 30 & 1,829 \\
\hline
\end{tabular}

obese, $67.7 \%$ were White, $29.4 \%$ also screened positive for depression, $41.9 \%$ engaged in risky drinking behavior, $36.6 \%$ used drugs, and $27.5 \%$ presented to the ED with GI complaints. The mean age of respondents who screened positive for an eating disorder was 17.1 ( $\mathrm{SD}=1.9)$.

Bivariate analyses between participants who had visited the $\mathrm{ED}$ at least once in the 12 months prior to their index visit and those who had not showed that those with prior ED use were more likely to be female $(\mathrm{OR}=1.3,95 \% \mathrm{CI}=1.0-1.6, p=.02)$ and $/$ or a recipient of public assistance $(\mathrm{OR}=1.8,95 \% \mathrm{CI}=$ 1.5-2.3, $p<.001)$ and were less likely to be White $(\mathrm{OR}=0.8,95 \% \mathrm{CI}=0.6-1.0, p=.03)$. Participants with prior ED visits were also significantly more likely to report drug use $(\mathrm{OR}=1.4,95 \% \mathrm{CI}=1.1$ $1.8, p=.004)$ and to screen positive for depression $(\mathrm{OR}=2.0,95 \% \mathrm{CI}=1.5-2.6, p<.001)$ and eating disorders (OR $=1.9,95 \% \mathrm{CI}=1.5-2.5, p<.001)$. There were no significant differences in past ED use by rates of risky drinking behavior or age (Table 1).

A $\chi^{2}$ test of participants who screened positive for an eating disorder and number of previous visits to the ED over the past 12 months was significant $\left(\chi^{2}\right.$ $=38.1, p<.001$ ). Of these respondents, $12.5 \%$ who had no previous visits to the ED prior to the date of the baseline survey screened positive for an eating disorder, whereas $43.3 \%$ who had visited the ED five or more times screened positive for an eating disorder (Table 2). To better understand the frequency of ED use, we estimated a negative binomial regression model using a count version of our outcome variable (outcome ranged from 0 to 14 previous visits).
This model revealed that patients who screened positive for an eating disorder have 1.6 times higher rate of visiting the ED than patients who do not screen positive for an eating disorder $(95 \% \mathrm{CI}=1.2-$ $2.1, p=.002$ ). Patients who screened positive for depression had a 1.8 times higher rate of ED use $(95 \% \mathrm{CI}=1.3-2.4, p<.001)$ than those who did not have depression, whereas recipients of public assistance had 1.7 times higher rate of ED use $(95 \% \mathrm{CI}=$ $1.3-2.2, p<.001)$ than nonrecipients.

When compared with the chief complaints of patients who screened negative for an eating disorder, a higher proportion of patients who screened positive presented with GI and/or GU chief complaints. Injury-related complaints were most common in those who screened negative for an eating disorder, whereas GI complaints were most common in those who screened positive. Of these patients, $27.5 \%$ who screened positive for an eating disorder, when compared with $21.2 \%$ of those who screened negative, presented with GI complaints, whereas $8.8 \%$, when compared with $5.4 \%$, presented with GU complaints. Binary analyses revealed that respondents who screened positive for an eating disorder were more likely to present with GI chief complaints $(\mathrm{OR}=1.4,95 \% \mathrm{CI}=1.1-1.9, p=.02)$ and GU chief complaints $(\mathrm{OR}=1.7,95 \% \mathrm{CI}=1.1-2.7, p=.03)$.

A logistic regression model estimated for a dichotomous chief complaint outcome $(1=\mathrm{GI}$ or GU chief complaint, $0=$ all other chief complaints) revealed that patients who screened positive for an eating disorder were 1.4 times more likely to present with GI or GU chief complaints than all other 
complaints $(95 \% \mathrm{CI}=1.0-1.8, p=.03)$. Of 246 patients, only one patient who screened positive for an eating disorder listed a complaint (bradycardia and worsening anorexia) that directly mentioned an eating disorder.

\section{Discussion}

This study examined the patterns of ED use among adolescents and young adults who screened positive for an eating disorder in comparison with those who did not. The results support our hypotheses that ED use would be increased among those with eating disorders and that the majority of visits would be due to complaints seemingly unrelated to the eating disorder. Patients who screened positive for an eating disorder were 1.6 times more likely to have visited the ED at least once in the year prior to their index visit when compared with patients who did not. Even more striking is that of all patients who had visited the ED frequently (five or more times in 12 months), $43.3 \%$ screened positive for an eating disorder. This is nearly three times higher than that would be expected based on the percentage of positive $(15.5 \%)$ versus negative $(84.5 \%)$ eating disorder screens. These findings are consistent with prior studies that have found increased rates of utilization of healthcare in multiple settings including primary care, mental health, and specialty services (gastroenterology and gynecology practices) in patients with eating disorders both prior to and following their diagnosis. ${ }^{1-5,7}$ However, few studies have focused specifically on the utilization of EDs by patients with eating disorders. Thus, the findings of the current study contribute important new information to the literature on healthcare utilization in eating disorders and, given the high costs associated with recurrent ED use, have important implications for the healthcare system as a whole. Although it is clear that specialized treatment for eating disorders would not be appropriate in the ED setting, screening and referral programs could be implemented. Screening programs that could identify patients with undiagnosed eating disorders and refer them for appropriate follow-up care may have the potential to reduce ED recidivism and related healthcare costs while simultaneously improving long-term outcomes for patients with eating disorders. Given the potential benefits of such programs, future research in this area may be warranted.

In addition to patients who screened positive for eating disorders, those who screened positive for depression and reported drug use, as well as those receiving public assistance, were significantly more likely to have visited the ED at least once in the year prior to their index visit. The findings in relation to depression and drug use are consistent with those from multiple prior studies that have shown increased utilization in all healthcare sectors by persons with mood and substance use disorders. ${ }^{29-}$ ${ }^{31}$ The increased ED use in recipients of public assistance is also consistent with prior research that has similarly demonstrated increased use in patients of lower socioeconomic status. ${ }^{32,33}$ This has been hypothesized by some to be due to inadequate access to primary care and other services by the uninsured or underinsured. ${ }^{33}$

In contrast to what has been reported in previous studies, this study found that patients who screened positive for risky drinking behavior were no more or less likely to have visited the ED in the year prior to their index visit. This is an unexpected finding given that much of the previous research on resource utilization related to alcohol use has found increased use in risky drinkers. ${ }^{29,31,34}$ One possible explanation for this is that our retrospective chart review assessed for prior visits to a single ED. The use of other healthcare services including nearby EDs and urgent care facilities would not have been detected, which may have resulted in an underestimation of the total number of actual ED visits. Additionally, risky drinking behavior is highly correlated with drug use in this study (41.9\% of patients who engage in risky drinking behavior also use drugs). This correlation may lessen the independent impact of risky drinking behavior on the rates of ED use. Finally, much of the prior research on rates of ED use in substance abusers has focused on adult patients, ${ }^{35,36}$ whereas the current study focuses younger patient population (average age 17.5 years). However, further research in this area is warranted.

In this study, the most frequently documented reasons for visits to the $\mathrm{ED}$ for patients who screened positive for an eating disorder were GIrelated complaints; however, injury-related complaints were most common in those who screened negative. In addition, those who screened eating disorder positive were more likely to present with GU complaints. These findings are consistent with earlier studies that showed an increased frequency of both GI- and GU-related complaints in patients with undiagnosed eating disorders. ${ }^{2,3,6}$ Of note, in our study, only one patient of the 246 patients who screened positive for an eating disorder reported a complaint that specifically mentioned an eating disorder. It is also important to note that the majority of patients who present to the University of Michigan Medical Center ED with exclusively psychiatric chief complaints such as depression or 
suicidal ideation are triaged directly to the Psychiatric Emergency Services and thus were not included in this screening study. Therefore, it is not surprising that we did not see high rates of psychiatric complaints amongst patients with eating disorders as had been found in other studies. ${ }^{2,5}$ It also makes the fact that $15.5 \%$ of all patients screened positive for an eating disorder in this population even more remarkable as these patients were primarily presenting for physical and not psychiatric complaints. These findings highlight the importance of screening for eating disorders in high-risk patients, such as those with frequent ED visits for GI- or GU-related complaints, regardless of their stated reason for the ED visit.

Other findings of this study are consistent from our prior study, which are reported and discussed elsewhere. ${ }^{14}$ Data used in the prior study were obtained only during the period of October 15, 2011 through March 1, 2012, whereas the current study included all data through September 14, 2011, which increased the sample size by 980 participants. The prevalence of eating disorders and their associations with female gender, increased BMI, risky drinking behavior, and depression remained consistent with our earlier findings, suggesting that these findings are reliable and reproducible amongst our patient population.

One association that did change in the current study was that eating disorder positive patients also were found to be significantly more likely to be receiving public aid than those who screened negative. In our prior study, this had been seen as a trend, but did not reach significance (likely due to the smaller sample size). While this may seem at first glance to be inconsistent with prior research on eating disorders, that is not the case. Although it is commonly believed that eating disorders are more prevalent among higher income groups, this belief has been repeatedly challenged over the past decade and is thought by many authors to be an artifact of the clinical samples used in earlier studies. ${ }^{37-39}$ Some more recent studies have shown that lower income groups may in fact be at increased risk of developing at least some types of eating disorders. $^{40,41}$

\section{Limitations}

Several potential limitations of this study warrant mentioning. First, the mixed cross-sectional and retrospective design of the study precludes its use as a means to determine causality.
In addition, there are several factors inherent to the study that may limit its generalizability. Of 4,153 patients who presented to the ED during the study period, only 2,679 (64.5\%) met inclusion criteria, and of these patients, 1,922 (86.4\%) completed the study for an overall participation rate of $46.3 \%$. Although no significant differences in baseline characteristics were noted amongst nonparticipants, they may have differed in undetected ways that could impact the study outcomes. In addition, the study examined patients in a single ED, which potentially limits its generalizability to other ED settings. Retrospective ED use was also only determined for this single site. It is possible that some patients may have utilized other EDs during this same time period and that this use would have been missed in this analysis. Finally, RAs were located only in the higher volume main ED to maximize their opportunities for patient recruitment. Because of the physical separation of the ED and the Psychiatric Emergency Services in our institution and the exclusion of patients in acute psychological distress, patients with primarily psychiatric complaints were mostly excluded from this study. Although this may limit generalizability to other patient populations, it is appropriate in this study which was intended to assess characteristics of ED use for patients with primarily medical complaints.

Additional limitations are related to the use of the modified SCOFF. This instrument has not been previously validated in the ED; however, findings for the number of patients with a positive SCOFF in the current study (15.5\%) are similar to those of the one prior study utilizing the tool in the ED $(16.0 \%),{ }^{14}$ suggesting that it performs consistently in this setting. Formal validation studies of this modified SCOFF are also needed to verify the effects of the modifications on its performance as a screening instrument. Such studies are currently being planned by the study team.

\section{Conclusion}

The rates of ED utilization among patients with eating disorders are significantly higher than the overall population of patients seeking ED care. Thus, healthcare providers in the emergency setting are uniquely positioned to play a key role in early recognition of patients with eating disorders. Subsequent referral to appropriate medical and psychological services could both reduce ED recidivism and lead to improved outcomes among these patients. Improved understanding of patterns of ED use in patients with eating disorder by the EM 
physician can help to facilitate their identification and referral. The findings of this study contribute significantly to this understanding by demonstrating that the majority of these patients will present with complaints not directly related to or recognizable as an eating disorder, particularly GI- and/or GU-related complaints. These findings highlight the importance of screening for eating disorders among patients with frequent ED use regardless of their stated reason for the visit.

The authors thank Carrie Smolenski, Linping Duan, MS, Sonia Kamat, and the patients and medical staff at the University of Michigan Medical Center for their support of this project.

M.A.W. and R.M.C. were responsible for the acquisition of data and are investigators on the grant funding this work. S.D.-H. and S.K.L. were responsible for the statistical analyses and wrote the initial draft of the manuscript. All authors provided critical feedback and revision of the manuscript. All authors contributed to and have approved the final manuscript.

\section{References}

1. Sansone R, Wiederman M, Sansone L. Healthcare utilization among women with eating disordered behavior. Am J Manag Care 1997;3:1721-1723.

2. Ogg E, Millar H, Pusztai E, Thom A. General practice consultation patterns preceding diagnosis of eating disorders. Int J Eat Disord 1997;22:89-93.

3. Johnson J, Spitzer R, Williams J. Health problems, impairment and illnesses associated with bulimia nervosa and binge eating disorder among primary care and obstetric gynaecology patients. Psychol Med 2001;31:1455-1466.

4. Mitchell J, Myers T, Crosby R, O’Neill G, Carlisle J, Gerlach S. Health care utilization in patients with eating disorders. Int J Eat Disord 2009;42:571-574.

5. Striegel-Moore R, DeBar L, Wilson G, Dickerson J, Rosselli F, Perrin $N$, et al. Health services use in eating disorders. Psychol Med 2008;38:1465-1474.

6. Mond J, Myers T, Crosby R, Hay P, Rodgers B, Morgan J, et al. Bulimic eating disorders in primary care: Hidden morbidity still? J Clin Psychol Med Settings 2010;17:56-63.

7. Emmanuel A, Stern J, Treasure J, Forbes A, Kamm M. Anorexia nervosa in gastrointestinal practice. Eur J Gastroenterol Hepatol 2004;16:1135-1142.

8. Becker A, Thomas J, Franko D, Herzog D. Disclosure patterns of eating and weight concerns to clinicians, educational professionals, family and peers. Int J Eat Disord 2005;38:18-23.

9. Striegel-Moore R, Dohm F, Kraemer H, Schreiber G, Crawford P, Daniels $S$. Health services use in women with a history of bulimia nervosa or binge eating disorder. Int J Eat Disord 2005;37:11-18.

10. Keski-Rahkonen A, Bulik CM, Neale B, Rose RJ, Rissanen A, Hoek HW, et al. Incidence and outcomes of bulimia nervosa: A nationwide population-based study. Psychol Med 2009;39:823-831.

11. Striegel-Moore R, Dohm F, Wilfley D, Pike K, Bray N, Krraemer $\mathrm{H}$, et al. Toward an understanding of health servises use in women with binge eating disorder. Obes Res 2004;12:799-806.
12. Treasure J, Russell G. The case for early intervention in anorexia nervosa: Theoretical exploration of maintaining factors. $\mathrm{Br}$ J Psychiatry 2011;199:5-7.

13. Mitchell J, Agras S, Wonderlich S. Treatment of bulimia nervosa: Where are we and where are we going? Int J Eat Disord 2007;40:95-101.

14. Dooley-Hash S, Banker J, Walton M, Ginsburg Y, Cunningham R. The prevalence and correlates of eating disorders among emergency department patients aged 14-20 Years. Int J Eat Disord, in press.

15. Gilbert E, Lowenstein S, Koziol-McLain J, Barta D, Steiner J. Chart reviews in emergency medicine research: Where are the methods? Ann Emerg Med 1996;27:305-308.

16. WHO. Physical status: The use and interpretation of anthropometry. Report of a WHO Expert Committee, WHO Technical Report Series 854. Geneva: World Health Organization, 1995.

17. Cotton M, Ball C, Robinson P. Four simple questions can help screen for eating disorders. J Gen Intern Med 2003;18:53-56.

18. Luck A, Morgan J, Reid F, O'Brien A, Brunton J, Price C, et al. The SCOFF questionnaire and clinical interview for eating disorders in general practice: comparative study. Br Med J 2002;325: 755-756.

19. Mond J, Myers T, Crosby R, Hay P, Rodgers B, Morgane J, et al. Screening for eating disorders in primary care: EDE-Q versus SCOFF. Behav Res Ther 2008;46:612-622.

20. Morgan J, Reid F, Lacey J. The SCOFF questionnaire: Assessment of a new screening tool for eating disorders. Br Med J 1999; 319:1467-1468.

21. Spitzer R, Kroenke K, Williams J. Validation and utility of a selfreport version of PRIME-MD; The PHQ primary care study. JAMA 1999;282:1737-1744.

22. Kroenke K, Spitzer R, Williams J. The patient health questionnaire-2 validity of a two-item depression screener. Med Care 2003;41:1284-1292.

23. Arroll B, Goodyear-Smith F, Crengle S, Gunn J, Kerse N, Fishman T, et al. Validation of PHQ-2 and PHQ-9 to screen for major depression in the primary care population. Ann Fam Med 2010;8:348-353.

24. Chung T, Colby S, Barnett N, Monti P. Alcohol use disorders identification test: Factor structure in an adolescent emergency department sample. Alcohol Clin Exp Res 2002;26:223-231.

25. Kelly T, Donovan J, Chung T, Bukstein O, Cornelius J. Brief screens for detecting alcohol use disorder among 18-20 year old young adults in emergency departments: Comparing AUDIT-C, CRAFFT, RAPS4-QF, FAST, RUFT-Cut, and DSM-IV 2Item Scale. Addict Behav 2009;34:668-674.

26. Reinert D, Allen J. The alcohol use disorders identification test: An update of research findings. Alcohol Clin Exp Res 2007;31: 185-199.

27. Dawson D, Grant B, Stinson F, Zhou Y. Effectiveness of the derived alcohol use disorders identification test (AUDIT-C) in screening for alcohol use disorders and risk drinking in the US general population. Alcohol Clin Exp Res 2005;29:844-854.

28. Babor T, WAW Group. The alcohol, smoking and substance involvement screening test (ASSIST): Development, reliability and feasibility. Addiction 2002;97:1183-1194.

29. Kessler R, Zhai S, Katz S, Kouzis A, Frank R, Edlund M, et al. Past-year use of outpatient services for psychiatric problems in the National Comorbidity Survey. Am J Psychiatry 1999;156: 115-123.

30. Stein M, O'Sullivan $\mathrm{P}$, Ellis $\mathrm{P}$, Perrin $\mathrm{H}$, Wartenberg A. Utilization of medical services by drug abusers in detoxification. J Subst Abuse 1993;5:187-193.

31. D'Onofrio G, Becker B, Woolard R. The impact of alcohol, tobacco, and other drug use and abuse in the emergency department. Emerg Med Clin North Am 2006;24:925-967. 
32. O'Brien G, Stein M, Zierler S, Shapiro M, O'Sullivan P, Woolard R. Use of the ED as a regular source of care: Associated factors beyond lack of health insurance. Ann Emerg Med 1997;30:286-291.

33. Hong R, Baumann B, Boudreaux E. The emergency department for routine healthcare: Race/Ethnicity, socioeconomic status, and perceptual factors. J Emer Med 2007;32:149-158.

34. Cherpitel C, Ye Y. Drug use and problem drinking associated with primary care and emergency room utilization in the US general population: Data from the 2005 national alcohol survey. Drug Alcohol Depend 2008;97:226-230.

35. Doupe M, Palatnick W, Day S, Chateau D, Sooden R, Burchill C, et al. Frequent users of emergency departments: Developing standard definitions and defining prominent risk factors. Ann Emerg Med 2012;60:24-32.

36. Sandoval E, Smith S, Walter J, Schuman S, Olson M, Striefler R, et al. A comparison of frequent and infrequent visitors to an urban emerency department. J Emerg Med 2010;38:115-121.
37. Gentile K, Raghavan C, Rajah V, Gates K. It doesn't happen here: Eating disorders in an ethnically diverse sample of economically disadvantaged, urban college students. Eat Disord 2007;15:405-425.

38. Wilfley D, Pike K, Dohm F, Streigal-Moore R, Fairburn C. Bias in binge eating disorder: How representative are recruited clinic samples? J Consult Clin Psychol 2001;69:383-388.

39. Smolak L, Striegel-Moore R, editors. Challenging the myth of the golden girl: Ethnicity and eating disorders. Eating Disorders: Innovative Directions in Research and Practice. Washington, DC: American Psychological Association, 2001.

40. Reagan P, Hersch J. Influence of race, gender, and socioeconomic status on binge eating frequency in a population-based sample. Int J Eat Disord 2005;38:252-256.

41. Shaw H, Ramirez L, Trost A, Randall P, Stice E. Body image and eating disturbances across ethnic groups: More similarities than differences. Psychol Addict Behav 2004;18:12-18. 